Topiques, études satoriennes

Topoï Studies, Journal of the SATOR

\title{
Introduction au volume 5 de Topiques (2020), Le Boire et le manger
}

\section{Yen-Maï Tran-Gervat}

Volume 5, 2021

Le manger et le boire dans la fiction narrative

URI: https://id.erudit.org/iderudit/1081519ar

DOI: https://doi.org/10.7202/1081519ar

See table of contents

Publisher(s)

SATOR, Société d'Analyse de la Topique Romanesque d'Ancien Régime

ISSN

2369-4831 (digital)

Explore this journal

Cite this document

Tran-Gervat, Y.-M. (2021). Introduction au volume 5 de Topiques (2020), Le Boire et le manger. Topiques, études satoriennes / Topoï Studies, Journal of the SATOR, 5. https://doi.org/10.7202/1081519ar viewed online.

https://apropos.erudit.org/en/users/policy-on-use/

This article is disseminated and preserved by Érudit.

Érudit is a non-profit inter-university consortium of the Université de Montréal, Université Laval, and the Université du Québec à Montréal. Its mission is to promote and disseminate research. 
Introduction au volume 5 de Topiques (2020),

Le Boire et le manger

Les colloques de la SATOR, dont celui qui est à l'origine de ce volume était le trente-deuxième de la série, sont toujours non seulement des rendez-vous scientifiques extrêmement stimulants, mais aussi des moments forts de sociabilité où, une fois par an, les membres de la SATOR anciens et nouveaux ont plaisir à se rencontrer et à se retrouver. Le colloque de 2018 avait lieu à l'automne, en plein "été des Indiens » (comme on dit au Québec un peu différemment de la chanson de Joe Dassin), à Geneva, New York, États-Unis, organisé par Catherine Gallouët, aujourd'hui co-éditrice de ce volume, et alors hôtesse hors pair, qui avait à cœur, à l'automne 2018, de faire découvrir aux Satoriens les richesses de cette région des Finger Lakes. Le thème du colloque s'était imposé de lui-même dès que le lieu en avait été arrêté : dans cette région rurale et gastronome, aux vignobles réputés, « Le Manger et le Boire » guideraient nos enquêtes topiques autant que nos activités annexes. Les participants ne furent pas déçus : entre les moments de réflexions sérieuses et conviviales sur les topiques alimentaires et culinaires, les palais furent gâtés lors des diverses pauses et déjeuners ; le traditionnel dîner de colloque fut organisé dans un restaurant installé au cœur d'une propriété vinicole et le séjour fut couronné par une excursion des plus plaisantes au milieu des vignobles des Finger Lakes, accompagnée par une dégustation des crus locaux. Le lieu avait donc idéalement rencontré la problématique, dont nous offrons un aperçu dans ce volume.

De l'espace politique (le mouvement fair trade) à l'espace social (justice alimentaire), le discours contemporain des Food Studies offre depuis quelques années une nouvelle interrogation du monde qui gagne peu à peu l'espace universitaire où il trouve naturellement sa place dans un échange pluridisciplinaire d'exploration et de communications entre des domaines aussi différents que l'anthropologie, l'économie, l'histoire, l'œnologie, la chimie, la philosophie... La SATOR invitait donc les chercheurs à un dialogue pluridisciplinaire à partir d'une thématique littéraire, le Manger et le Boire. Comment et en quoi la production littéraire reflète-t-elle l'importance vitale du Manger et du Boire ? Quelle place et quelles fonctions occupent la nourriture et la boisson dans la littérature narrative ?

Les neuf articles ici retenus répondent chacun à sa manière à ces questions et permettent d'explorer le Manger et le Boire dans des corpus de différentes littératures et cultures, à des époques variées.

Nous les avons groupés en deux parties distinctes et complémentaires. Les quatre articles de la première partie sont des invitations à une dégustation « horizontale » des topoi explorés, comme on déguste un même vin sur plusieurs millésimes: les corpus en sont en effet diachroniques. Monique Moser-Verrey étudie « Le cœur mangé à plusieurs sauces » du MoyenÂge à la fin du XVIII ${ }^{\mathrm{e}}$ siècle : le mari jaloux donnant à dévorer à son épouse ignorante le cœur de son amant est en effet une configuration narrative récurrente dont l'article étudie les structures et les variantes. Juan Ibeas et Lydia Vazquez, dans leur article « Représentations du corps ivre dans la littérature et la peinture hispaniques », examinent des textes et des tableaux réalisés en Espagne entre le XIIII ${ }^{\mathrm{e}}$ siècle et le début du XIX ${ }^{\mathrm{e}}$ siècle et montrent notamment la 
manière dont s'y reflètent les principaux discours sur le vin et ceux qui le consomment, entre éloge de la vitalité et condamnation de l'excès. Sans quitter la péninsule ibérique, Constança Vieira de Andrade propose, dans un article en anglais, d'examiner la place des relations alimentaires dans les récits de la rencontre avec l'étranger dans la littérature portugaise, du XV au $\mathrm{XXI}^{\mathrm{e}}$ siècle. C'est le même point de départ chronologique que choisit Catherine Gallouët, ancrant son étude sur les topoi du cannibalisme dans les textes des premiers Européens en Amérique : elle montre bien la manière dont, quasi à l'identique, le mythe du sauvage cannibale migre de l'Amérique du XVe siècle vers l'Afrique du XVIIIe siècle pour finir par être associé aux Noirs dans les récits de voyage comme dans les fictions ou illustrations.

Les cinq articles de la seconde partie proposent pour leur part à chaque fois une « dégustation verticale ", avec à chaque fois l'examen détaillé des topoi proposés dans une œuvre donnée ou une période spécifique. Dans son article « Des mets et des mots : nommer les mets dans la littérature espagnole du XVIe siècle », Nathalie Peyrebonne renforce la présence des littératures hispaniques au sein du volume en explorant des configurations lexicales récurrentes ou singulières suscitées par l'évocation des mets variés de la Renaissance espagnole. Martine Debaisieux, pour sa part, nous invite « À la table d'Apollon », pour examiner en détails la manière dont le manger et le boire évoluent « de l'abondance à la modération », dans L'Histoire comique de Francion de Sorel (1623). L'exploration du XVII ${ }^{\mathrm{e}}$ siècle français prend un tour inattendu et néanmoins fort pertinent, lorsque Nicolas Pelleton propose d'étudier les enjeux de la nourriture (certes à la fois terrestre et céleste) chez Bossuet panégyriste. L'article d'Elodie Ripoll est consacré au XVIII ${ }^{\mathrm{e}}$ siècle et aux textes de fiction narrative qui, au cours de cette période, contribuent à l'élaboration d'une poétique du chocolat, boisson énergisante et parfois dangereuse. Enfin, Suzan Van Dijk met en dialogue la correspondance et les romans d'Isabelle de Charrière pour examiner les convergences ou divergences entre biographie et fiction quand il est question de nourriture et d'alimentation.

Alors que les chercheuses et chercheurs du monde entier attendent avec impatience le moment où ils pourront retrouver la convivialité de vrais colloques, nous sommes heureuses de donner à lire quelques-uns des morceaux de choix du colloque SATOR, cru 2018 : bonne dégustation !

Yen-Maï Tran-Gervat 